\title{
On determining the undrained bearing capacity coefficients of variation for foundations embedded on spatially variable soil
}

https://doi.org/10.2478/sgem-2019-0037

received December 27, 2019; accepted February 19, 2020.

\begin{abstract}
This paper presents an efficient method and its usage for the three-dimensional random bearing capacity evaluation for square and rectangular footings. One of the objectives of the study is to deliver graphs that can be used to easily estimate the approximated values of coefficients of variations of undrained bearing capacity. The numerical calculations were based on the proposed method that connects three-dimensional failure mechanism, simulated annealing optimization scheme and spatial averaging. The random field is used for describing the spatial variability of undrained shear strength. The proposed approach is in accordance with a constant covariance matrix concept, that results in a highly efficient tool for estimating the probabilistic characteristics of bearing capacity. As a result, numerous three-dimensional simulations were performed to create the graphs. The considered covariance matrix is a result of Vanmarcke's spatial averaging discretization of a random field in the dissipation regions to the single random variables. The matrix describes mutual correlation between each dissipation region (or between those random variables). However, in the presented approach, the matrix was obtained for the expected value of undrained shear strength and keep constant during Monte Carlo simulations. The graphs were established in dimensionless coordinates that vary in the observable in practice ranges of parameters (i.e., values of fluctuation scales, foundation sizes and shapes). Examples of usage were given in the study to illustrate the application possibility of the graphs. Moreover, the comparison with the approach that uses individually determined covariance matrix is shown.
\end{abstract}

Keywords: Spatial averaging; random fields; random bearing capacity; fluctuation scale; failure mechanism.

* Corresponding author: Marcin Chwata, Wroclaw University of Science and Technology, Wybrzeże Wyspiańskiego 27, 50-370 Wroctaw, Poland, E-mail: marcin.chwala@pwr.edu.pl

\section{List of Symbols}

$\mathrm{BC}$ - bearing capacity

$\mathrm{CPT}$ - cone penetration test

SOF - scale of fluctuation

$b$ - foundation width

$a$ - foundation length

$\theta_{v}$ - vertical scale of fluctuation

$\theta_{h}$ - horizontal scale of fluctuation

\section{Introduction}

Incorporating spatial variability of soil and rock masses has recently become an important subject for geotechnical engineers' interest. In recent times, probabilistic approaches are being used in many fields of geotechnical engineering like tunnelling (e.g., Lu et al., 2018; Pan and Dias, 2018; Chen et al., 2019) or deep excavations (e.g., Ching et al., 2017; Goh et al., 2019). Popularity of probabilistic approaches is also visible in the case of shallow foundations bearing capacity estimations. The direct reason for this interest is the popularity of cone penetrometer test soundings (CPT), and consequently, obtaining enough data to estimate the vertical scale of fluctuation (that characterized the considered soil strength spatial variability). On the other hand, the recent development and increase of experience in the field of estimating fluctuation scales techniques accelerate the development towards the use of random field theory to assess bearing capacity (Ching et al., 2018). However, most of the existed studies on this subject use twodimensional simplification of the problem by assuming plane strain conditions (e.g., Fenton and Griffiths 2008; Pieczyńska-Kozłowska et. al., 2015; Ali et. al., 2016, Johari et al., 2017; Puła and Chwała 2018). Due to the ignorance of soil spatial variability in one direction (perpendicular to the assumed plane), this simplification may cause conservative estimation of variation coefficient of bearing capacity. To avoid this effect, it is necessary to perform 


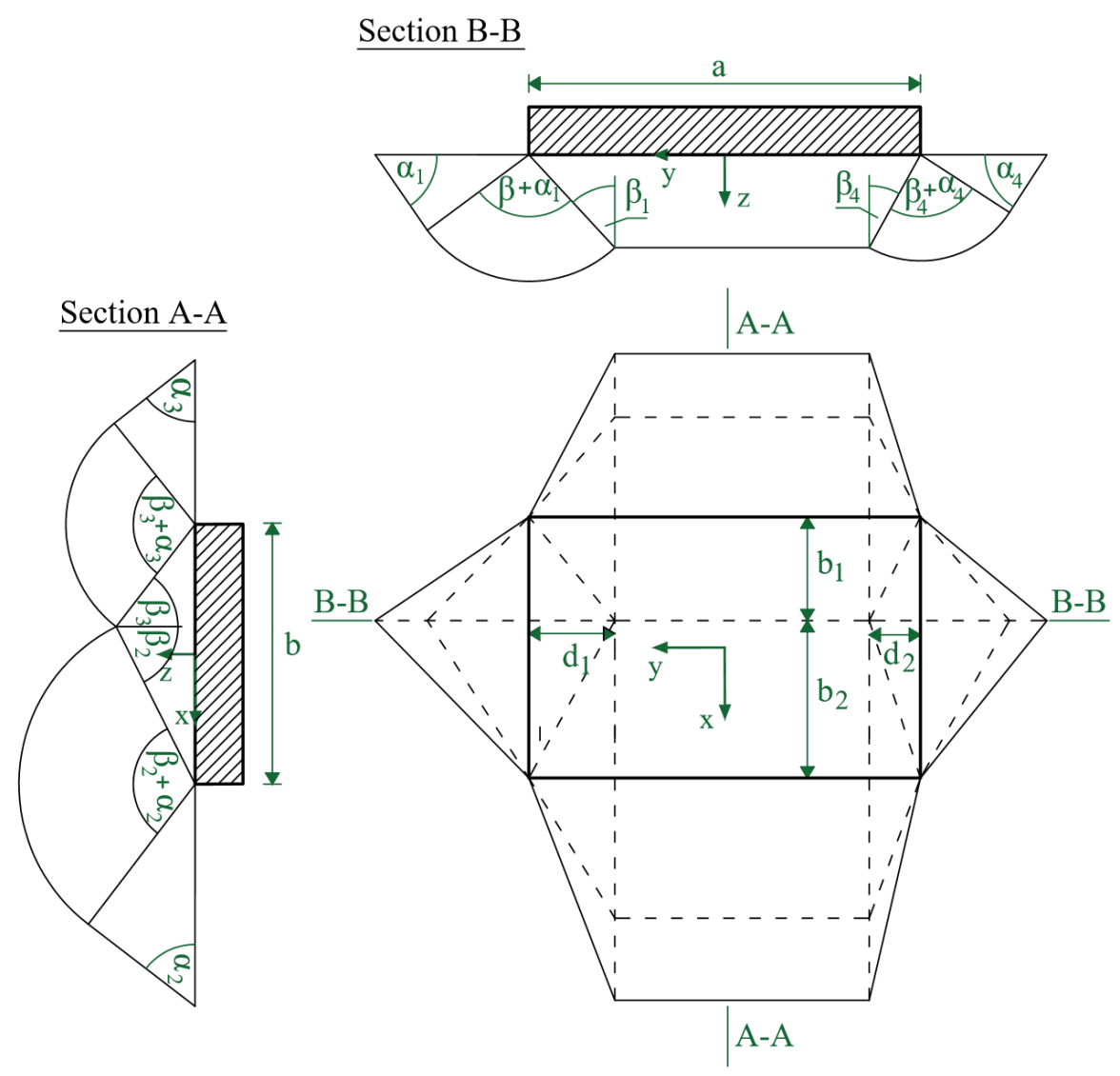

Figure 1: Failure geometry for a rough foundation base in the case of rectangular foundation. More details on failure geometry can be found in Chwała (2019a).

numerical analyses in three dimensions. However, the three-dimensional analyses in the case of a finite element approach require a lot of computational effort. As a result, there is a lack of such analyses in the literature, one of the first papers in this area was published by Kawa and Puła (2019) and uses the finite difference method. Contrary to the finite element method approaches, the method proposed by Puła and Chwała $(2015,2018)$ that uses conjunction of Vanmarcke's spatial averaging (1983) and kinematical failure mechanisms may result in significantly improved computational efficiency. The application of this approach to the three-dimensional issue of bearing capacity estimation of square or rectangular foundations in the case of undrained conditions was developed recently by Chwała (2019a). In this study, the above-mentioned method is modified in a way to ensure further increase in computational efficiency. The proposed modification uses the constant covariance matrix instead of individually determined as in Chwała (2019a). The constant covariance matrix is received by using the expected values of soil strength parameters. Therefore, in the case of this study, an expected value of undrained shear strength is used. The covariance matrix is determined based on the optimal failure geometry that is the result of optimization procedure. Due to the usage of upper bound analysis, the geometry for the specified undrained shear strength is searched in a way to minimize the resulting bearing capacity. The above briefly described method was used for analysing numerous scenarios to enable the creation of graphs that allow an immediate read of the approximate value of the variation coefficient of undrained bearing capacity (BC). In the proposed method, as the prerequisites, the undrained shear strength mean value $\left(\mu_{c_{u}}\right)$, standard deviation $\left(\sigma_{c_{u}}\right)$ and vertical fluctuation scale $\left(\theta_{v}\right)$ are needed. However, all this information can be obtained based on the soil sounding (e.g., Pieczyńska-Kozłowska et al., 2017; Nuttall, 2019). As a result of the numerical analyses, a comprehensive behaviour of variation coefficient of undrained $\mathrm{BC}$ in a wide range of foundation geometries and fluctuation 
scales are demonstrated on the created graphs. They can be used in the practical applications to estimate the $\mathrm{BC}$ coefficient of variation or as a comparison for other probabilistic methods.

\section{Numerical algorithm}

\subsection{Failure geometry optimization}

In this study, a Prandtl-type three-dimensional failure mechanism for a rough foundation base is assumed (Gourvenec et al, 2006). However, for the purpose of this study, a probabilistic version of the failure mechanism is needed (to calculate the bearing capacity in the case of different values of undrained shear strength are applicate to different dissipation regions). Therefore, the results obtained in the study by Chwała (2019a) were used as a basis for the proposed approach and numerical analyses performed in this study. The geometry of the failure mechanism is shown in Fig 1.

A set of failure geometry parameters shown in Fig. 1 is responsible for describing failure geometry. However, for unique failure geometry determination, only eight of those parameters are necessary, namely: six angles $\alpha_{1}, \alpha_{2}$, $\alpha_{3}, \alpha_{4}, \beta_{2}$ and $\beta_{3}$; and two lengths $d_{1}$ and $d_{2}$. Additionally, the foundation width and length are also necessary, but those two parameters are not a subject of optimization (they are constant during the optimization process). The resulting bearing capacity formula is given in Appendix A. The selected set of failure geometry parameters is subjected to changes during the optimization process of finding the minimum bearing capacity. Note that the bearing capacity formula is the objective function. The optimization method is based on the simulated annealing (Kirkpatrick et al., 1983; Kirkpatrick, 1984). The objective of using simulated annealing is to overcome local minima in the bearing capacity function. The simple gradient method can be more efficient than simulated annealing approach, but not resisted on avoiding local minima. On the other hand, the optimization issue has eight degrees of freedom, and consequently, direct simulation optimization based on Monte Carlo are not applicable due to poor efficiency. The bearing capacity formula and optimization method for the selected failure mechanism were discussed in Chwała (2019a) and the details are not repeated here. Generally, the procedure during the simulation uses a so-called acceptance probability $P_{a}$ that is intended to accept or reject a subsequent solution (that is obtained by small changes in the failure geometry

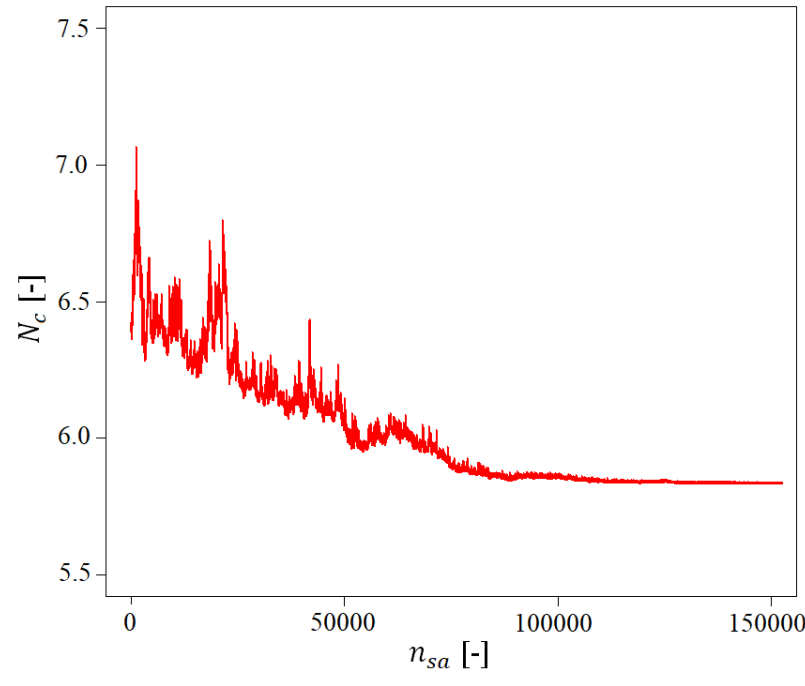

Figure 2: Value of bearing capacity during simulation within optimization procedure

parameters). However, the ability to overcome local minima requires that during simulation, the solutions characterized by higher bearing capacity (worse solution) can be accepted. The value of $P_{a}$ is in decreasing tendency during the simulation, and approaches almost zero at the end of simulation. As a result of using the above briefly described method, the approximate location of a global minimum of the bearing capacity function is found together with the corresponding optimal failure geometry. One exemplary optimization is demonstrated in Fig. 2. As shown there, the ability of the optimization scheme to avoid local minima is clearly visible.

\subsection{Spatial averaging}

The kinematical failure mechanism can be used in conjunction with random field theory by using Vanmarcke's (1983) spatial averaging. By this procedure, the average values of undrained shear strength are determined for each dissipation region resulting from failure geometry. The application of spatial averaging provides no necessity of direct random field sample generation. Instead of that, the averaged values of undrained shear strength are generated. The generation takes place from single random variables obtained after discretization of the initial random field. Each random variable is dedicated to the corresponding dissipation region. Therefore, in this study, there are 30 random variables (because the failure mechanism consists of 30 dissipation regions). However, those variables are not independent, they are correlated in accordance with 
their relative positions, assumed correlation structure and values of fluctuation scales. Each random variable is obtained by integrating over the dissipation region geometry; thus, its shape, size and position with respect to the coordinate system is taken into account. To obtain full information about correlation between each of those 30 random variables, the matrix of size 30 over 30 is necessary. This matrix is called as the covariance matrix and denoted as $C_{X}$. The general formula that constitutes spatial averaging can be expressed as in Eq. (1):

$$
X_{V}=\frac{1}{|V|} \iiint X(x, y, z) \mathrm{d} x \mathrm{~d} y \mathrm{~d} z
$$

where, $V$ is the domain of averaging that is related to the specified dissipation region, $X$ is the initial random field (before averaging) and $X_{V}$ is the random variable obtained after spatial averaging proper to the averaging domain $V$. In this study, a stationary lognormal random field is assumed as initial random field. As a result of averaging, the mean value of $X_{V}$ is preserved; however, the variance of $X_{V}$ is subjected to reduction. The new variances and covariances can be determined from Eq. (2) (see Puła, 2007; Puła and Chwała, 2015):

$$
\begin{gathered}
\operatorname{Cov}\left(X_{i}, X_{j}\right)=\frac{1}{\left|V_{i}\right|\left|V_{j}\right|} \int_{V_{i}} \int_{V_{j}} R\left(x_{i}, y_{i}, z_{i}, x_{j}, y_{j}, z_{j}\right) \\
d V_{i}\left(x_{i}, y_{i}, z_{i}\right) d V_{j}\left(x_{j}, y_{j}, z_{j}\right)
\end{gathered}
$$

where, indices $i$ and $j$ denote dissipation regions; $i$, $j=1, \ldots, 30$. Function $R$ is a covariance function. In this study, a Gaussian covariance function is assumed (e.g., Fenton and Griffiths, 2008); moreover, the correlation structure is assumed to be separable in each direction. As a result, the covariance function given in Eq. (3) is used:

$$
\begin{gathered}
R(\Delta x, \Delta y, \Delta z)=\sigma_{c_{u}}^{2} \exp \left\{-\left[\left(\sqrt{\pi} \frac{\Delta x}{\theta_{x}}\right)^{2}+\right.\right. \\
\left.\left.+\left(\sqrt{\pi} \frac{\Delta y}{\theta_{y}}\right)^{2}+\left(\sqrt{\pi} \frac{\Delta z}{\theta_{z}}\right)^{2}\right]\right\}
\end{gathered}
$$

where, $\Delta x, \Delta y, \Delta z$ are distances between points in $x, y$ and $z$ direction, respectively. Parameters $\theta_{x}, \theta_{y}$ and $\theta_{z}$ are fluctuation scales in $x, y$ and $z$ direction, respectively. In this study, both horizontal fluctuation scales are assumed to be equal, $\theta_{x}=\theta_{y}$, and denoted later as horizontal fluctuation scale $\theta_{h}$. Analogously, $\theta_{z}$ is called as vertical fluctuation scale and denoted as $\theta v$. By substituting the same index values in Eq. (2) instead of covariance, the formula for variance is obtained. Note that, for the considered scenario, there are $30 \times 30=900$ possible combinations. As a result, the covariance matrix has 900 components; however, due to the symmetricity of the matrix, there are 465 unique components. The derivation of the covariance matrix components is demonstrated in the earlier mentioned paper by Chwała (2019a). Despite different approaches developed there, those formulas can by successively utilized in this study. In the paper by Chwała (2019a), the covariance matrix was determined individually for each Monte Carlo simulation. However, in this study, a different approach is proposed; namely, the concept of constant covariance matrix. It means that the covariance matrix is kept constant during Monte Carlo simulation. Nevertheless, the important question arises here: which covariance matrix should be selected for this application? A natural choice is to use a covariance matrix obtained for the failure geometry established for the expected value of undrained shear strength. This choice was first applicable for the classic Prandtl failure mechanism by Puła (2007). The determination of the covariance matrix is the most time-consuming element in the proposed numerical algorithm. For example, if one thousand Monte Carlo simulations have to be performed to estimate some probabilistic characteristics of bearing capacity, in the case of constant covariance matrix, only one matrix determination is needed instead of one thousand, as in the algorithm given in Chwała (2019a). This significantly reduced the overall time that is needed for numerical analyses. As detailed in the introduction section, to achieve the objective of this study, numerous simulations are needed. Therefore, the constant covariance matrix is assumed to make them possible to calculate in a rational period of time. As shown in the next section, the impact of this assumption is very limited and is acceptable for the planned applications.

\subsection{Method}

The numerical algorithm that uses the constant covariance matrix is described below. The algorithm consists of two main parts. The first one is the determination of the covariance matrix that takes place before the Monte Carlo loop started. The second part is the actual Monte Carlo loop with the generation of averaged undrained shear strengths and bearing capacity calculation. Below, the detailed algorithm is described. Moreover, the flow chart of the procedure is shown in Fig. 3. 
Step 1. Determines the constant covariance matrix. The covariance matrix is determined basing on the optimal failure geometry that was obtained for the expected value of the undrained shear strength. At this stage, the same value of $c_{u}$ is assumed in all dissipation regions. In this particular case, the failure geometry has two planes of symmetry (not like in Fig. 1, where the general nonsymmetrical case is shown). The covariance matrix $C_{X}$ is kept constant during the simulation process. Set $k=1$.

Step 2. Begins the Monte Carlo simulation. If $k>N$, go to step 6 . If not, go to step 3 ( $N$ is the assumed number of repetitions).

Step 3. Generates a vector of 30 normal independent components. Each component describes undrained shear strengths $c_{Y u, i}$ Thegeneration process uses a pseudo-random number generator. As the probabilistic characteristic, the initial random field parameters transformed to underlying normal distribution are assumed $\left(\mu_{Y c_{u}}\right.$ and $\left.\sigma_{Y c_{u}}^{2}\right)$. The transformation is shown in Eq. (4).

$$
\begin{gathered}
\sigma_{Y c_{u}}^{2}=\ln \left(1+\frac{\sigma_{c_{u}}^{2}}{\mu_{c_{u}}^{2}}\right) \\
\mu_{Y c_{u}}=\ln \left(\mu_{c_{u}}^{2}\right)-0.5 \sigma_{Y c_{u}}^{2}
\end{gathered}
$$

As a result of the generation, the following vector of 30 components is obtained: $\left(c_{Y u, 1}, \ldots, c_{Y u, 30}\right)$.

Step. 4. Computes the averaged undrained shear strengths. The averaged undrained shear strengths are determined by the algorithm proposed by Puła and Chwała (2015). This procedure uses Cholesky decomposition of the covariance matrix $C_{X}$ (Horn, 1985). However, before the decomposition, the covariance matrix is transformed from a lognormal covariance matrix to the normal one. The resulting triangular matrix is multiplied by the standardized vector of the independent normal vector obtained in step 3. Finally, the resulting correlated undrained shear strengths are transformed again to the lognormal distribution. As a result of this procedure, the final vector of the averaged undrained shear strengths is obtained $\left(\bar{c}_{u, 1}, \ldots, \bar{c}_{u, 30}\right)$. The covariance matrix used in this step was determined in step 1.

Step 5. Calculates the bearing capacity corresponding to the undrained shear strengths $\left(\bar{c}_{u, 1}, \ldots, \bar{c}_{u, 30}\right)$. The bearing capacity $p_{N}$ is determined by using the optimization procedure described in section 2.1. Set $k=k+1$ and go to step 2.

Step 6. Calculates the probabilistic characteristics of bearing capacity. The formulas for mean value, standard

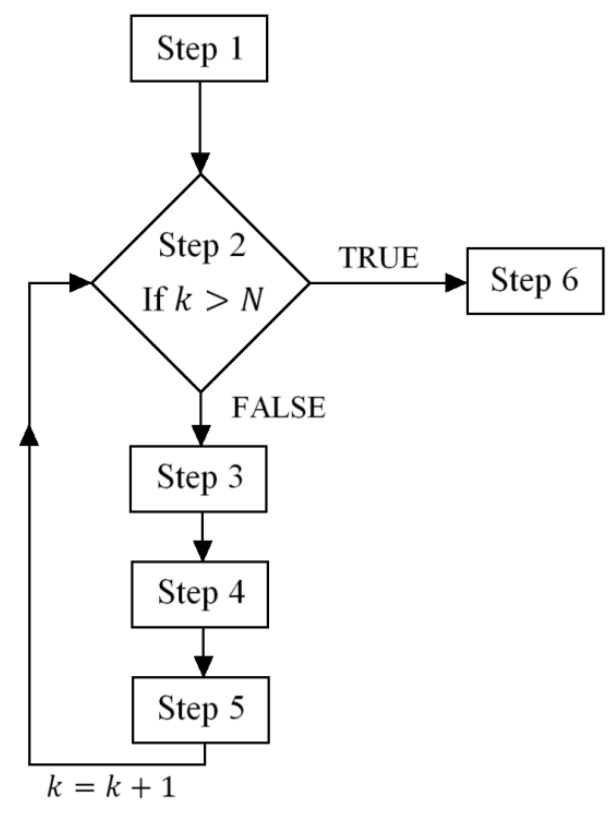

Figure 3: Flow chart of the numerical algorithm. Detailed descriptions of the steps are in the text.

deviation, and coefficient of variation of $\mathrm{BC}$ are shown in Eq. (5), Eq. (6) and Eq. (7), respectively.

$$
\begin{gathered}
\mu_{p}=\frac{1}{N} \sum_{i=1}^{N} p_{i} \\
\sigma_{p}=\sqrt{\frac{\sum_{i=1}^{N}\left(p_{i}-\mu_{p}\right)}{N-1}} \\
\operatorname{CoV}_{p}=\frac{\sigma_{p}}{\mu_{p}}
\end{gathered}
$$

End of an algorithm.

\section{Numerical accuracy}

The concept of using a constant covariance matrix may introduce differences in the obtained random bearing capacity estimations in comparison with the situation where the covariance matrix is determined individually to the generated undrained shear strengths. However, earlier experiences in the case of two dimensional Prandtl's failure mechanism indicated that those differences are very small, for more details please see Puła and Chwała (2015). Despite this, for the purpose of this study, the comparison analyses are conducted to verify the impact 
Table 1: Comparison of the results obtained in this study with those obtained by Simoes et al. (2014) by random finite limit analysis.

\begin{tabular}{lllll}
\hline Scenario & Method & $\mu_{N_{c}}[-]$ & $\sigma_{N c}[-]$ & COV $_{N c}[-]$ \\
\hline $\operatorname{COV}_{c_{u}}=0.5$ & RFLA (Simoes et al. 2014) & 4.77 & 1.68 & 0.35 \\
$\theta=8.0 \mathrm{~m}$ & This study & 5.19 & 2.14 & 0.41 \\
$\operatorname{COV}_{c_{u}}=1.0$ & RFLA (Simoes et al. 2014) & 3.72 & 1.23 & 0.33 \\
$\theta=2.0 \mathrm{~m}$ & This study & 4.51 & 1.77 & 0.39 \\
\hline
\end{tabular}

on accuracy for the three-dimensional case. For this reason, a series of numerical analyses were performed to examine the variation coefficients of the undrained bearing capacity obtained by the standard version of the algorithm (Chwała, 2019a) and by the approach presented in this study. The analyses cover variety of foundation lengths; namely, $a=1.0 \mathrm{~m}, a=3.0 \mathrm{~m}, a=5.0 \mathrm{~m}$ and $a=10.0$ $\mathrm{m}$; the foundation width is assumed constant, $b=1.0 \mathrm{~m}$. Variety of fluctuation scales are examined; however, in this comparison the isotropic correlation structure is assumed $\left(\theta_{h}=\theta_{v}=\theta\right)$. The results are shown in Fig. 4. It is clearly visible that the impact of using constant covariance matrix in the case of the three-dimensional analyses is very limited. Moreover, it is near the numerical accuracy connected with the finite number of Monte Carlo simulations used to estimate coefficient of variation of undrained bearing capacity. In the analysed scenarios, a number of $N=2000$ simulations were used.

In Fig. 4, the results obtained by random finite limit analysis are also shown for the same soil conditions. Those results were obtained by Huang et al. (2013) for plane strain conditions (see green dashed line in Fig. 4); therefore, they cannot be compared directly with those obtained by the approach proposed in this study. Nevertheless, Fig. 4 illustrates the importance of considering spatial variability in three dimensions - for longer foundations, plane strain assumption may provide more conservative $\mathrm{BC}$ coefficient of variation estimates.

Moreover, by using the algorithm proposed in this study, the same scenarios were examined as in the study by Simoes et al. (2014), where the three dimensional spatial variability was considered for $8 \mathrm{~m}$ foundation section (the authors used random finite limit analysis). Note that in the study by Simoes et al. (2014) plane strain conditions were assumed. Therefore, the obtained failure mechanism was not fully three-dimensional; however, due to its proportions $8.0 \mathrm{~m}$ over $1.0 \mathrm{~m}$, this impact is not very significant. The comparison is shown in Table 1 . The results obtained by both approaches are consistent; however, in the study by Simoes et al. (2014), lower

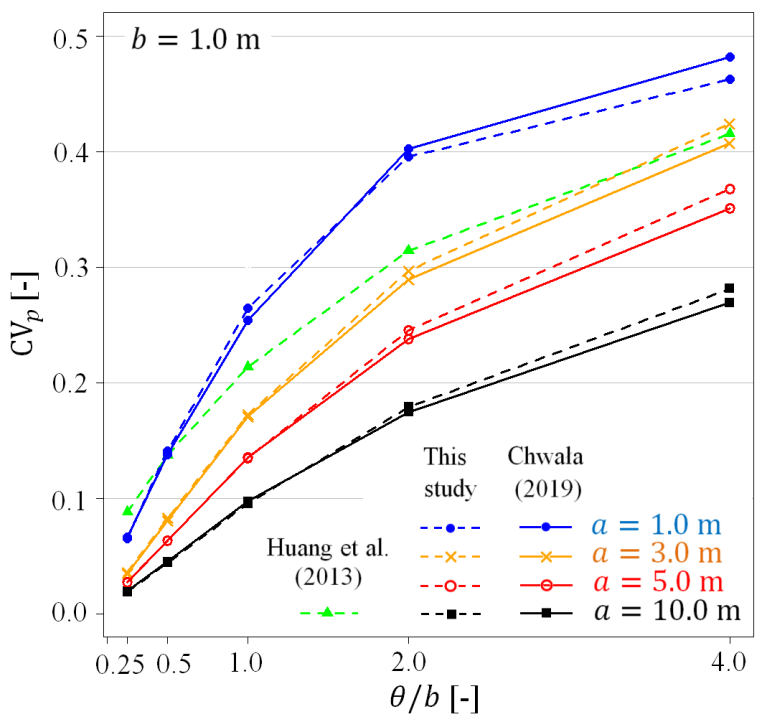

Figure 4: Comparison of bearing capacity variation coefficients obtained by the method proposed in this study (constant covariance matrix) with standard algorithm (individual covariance matrix, Chwała (2019a)). The relative differences between both approaches are below $5 \%$, which is sufficient for the purpose of this study. A detailed description is in the text.

bearing capacity mean values and standard deviations were obtained. Certain parts of these differences can be explained by the fully three-dimensional mechanism considered in this paper and different covariance functions assumed in both studies.

As mentioned above, a number of 2000 simulations was used for the estimation of bearing capacity mean value and standard deviation. This number is found to be sufficient for the objectives of this study. To illustrate that the mean value and standard deviations are stabilized for $N=2000$, in Fig. 5, those parameters together with coefficient of variation of bearing capacity for one particular simulation are shown. It can be observed that for $N>500$, the concerning values converged. For the application needed in this study, the obtained accuracy is sufficient. 


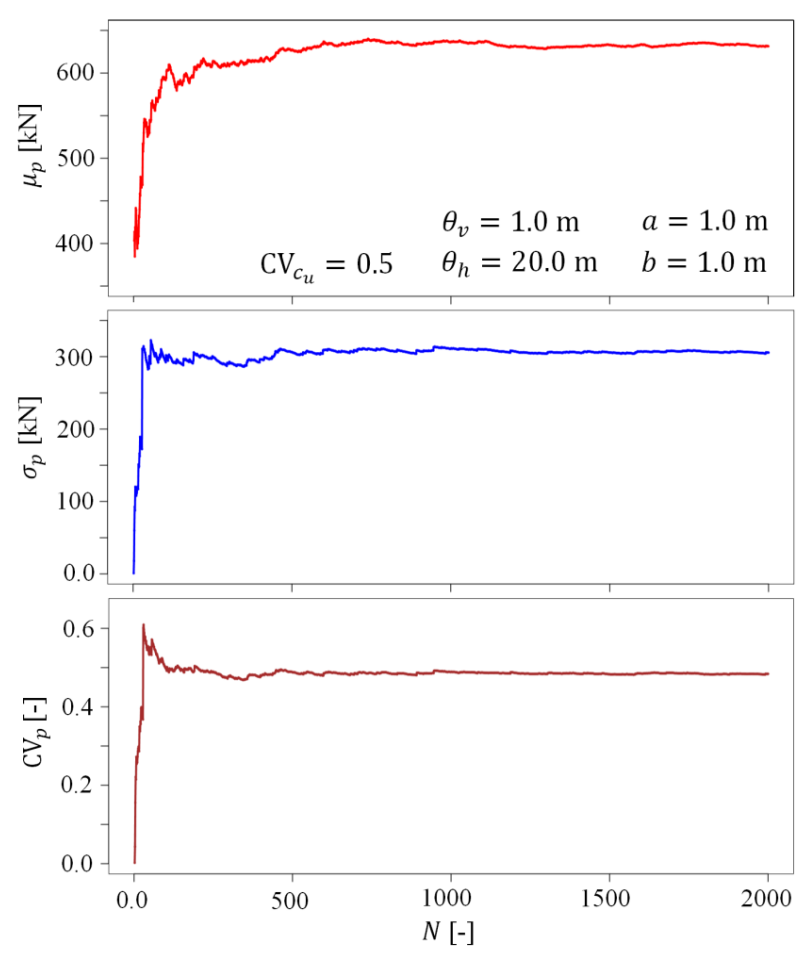

Figure 5: Mean value, standard deviation and variation coefficient of bearing capacity as a function of simulation number $\boldsymbol{N}$. The square foundation is assumed with geometry parameters and fluctuation scales detailed in the figure.

\section{Numerical analyses}

To construct graphs that are sufficiently accurate for the approximate estimation of the coefficient of variation of undrained bearing capacity in a wide range of soil and foundation geometry scenarios, numerous numerical simulations have to be performed. The results are present in a dimensionless coordinate system to cover almost all possible scenarios (from practical application viewpoint in the case of standard foundation sizes). As the vertical axis, the dimensionless coefficient of variation of undrained bearing capacity $D_{p}$ defined as $D_{p}=\mathrm{COV}_{p} / \mathrm{COV}_{c_{u}}$ ) is assumed in the graphs. As a result of such a definition, $D_{p}$ takes values from 0.0 to 1.0. Note that if the undrained shear strength be described by a single random variable (instead of random field like in this study), the corresponding $D_{p}$ value will be equal to 1.0. However, by introducing spatial averaging of undrained shear strength, $D_{p}$ is bounded by the above-mentioned range. As a horizontal axis, a ratio between foundation length and horizontal fluctuation scale is assumed, $a / \theta_{h}$. Due to the wide range of quotient $a / \theta_{h}$ the logarithmic scale is assumed for the horizontal axis. In this coordinate system, a series of results for a variety of $\theta_{v} / b$ ratios and $b / \theta_{h}$ ratios were plotted. To use these charts, the information of foundation size ( $a$ and $b$ ), coefficient of variation of $c_{u}$ and vertical fluctuation scale are needed. This set of values is necessary in the case of practical applications because $\operatorname{COV}_{c_{u}}$ and $\theta_{v}$ can be quite easily determined basing on CPT sounding (PieczyńskaKozłowska, 2015). The estimation of horizontal fluctuation scale is still a challenging task; however, for the conservative estimation of $\mathrm{COV}_{p}$, the infinite value of $\theta_{h}$ can be assumed. To prepare the charts given in the following section, 155 scenarios were analysed in total. This number of scenarios results in $3.1 \times 10^{5}$ performed numerical simulations. The analyses were conducted for $\theta_{v} / b=2.0,1.0,0.75,0.5,0.25$ and 0.125 . For specified $\theta_{v} /$ $b$ different $b / \theta_{h}$ are assumed; $b / \theta_{h}=0.1,0.2,0.5,1.0,2.0$, 4.0, 8.0. However, due to the requirements that foundation length $a \geq b$, not all values of $b / \theta_{h}$ are admissible (this can be seen in the charts shown in the following section). Finally, for each considered $b / \theta_{h}$, a series of $a / \theta_{h}$ are examined. All performed analyses are for square and rectangular foundations, other foundation shapes are not considered in this study. The surface foundation was assumed.

\section{Results}

The resulting coefficients of variation of undrained bearing capacity for the scenarios described in the previous section are used here to construct the graphs for reading approximate values of undrained bearing capacity (without the necessity of conducting numerical calculations). The description of the graphs is given below; however, the examples of their application are shown in section 5.1. The final results were divided into two graphs shown in Fig. 6 and Fig. 7. This separation is to ensure their clarity. From Fig. 6, the values of coefficients of variations for ratios $\theta_{v} / b \in[2.0,1.0]$ and $\theta_{v} / b \in[0.25$, $0.125]$ can be read. However, from Fig. 7, the coefficients of variations for ratios $\theta_{\sqrt{ }} b \in[1.0,0.25]$. Note that vertical axes in Fig. 6 and Fig. 7 are a dimensionless ratio of $D_{p}=\mathrm{COV}_{p}$ $/ \mathrm{COV}_{c_{u}}$ (this ratio expresses the reduction magnitude of $\mathrm{COV}_{c_{u}}{ }_{u}$; ; therefore, to calculate a coefficient of variation of undrained shear strength, the read value of $D_{p}$ has to be multiplied by the coefficient of variation of undrained shear strength $\operatorname{COV}_{c_{u}}$. As shown in Fig. 6 and Fig. 7, the values of $D_{p}$ decrease with a decrease in $\theta_{v} / b$ ratio. Please note that the left edges of all lines represent the square foundation, for example, in Fig. 6 , for $\theta_{v} / b=0.25$ and $b / \theta_{h}=0.1$ on the left edge, $a / \theta_{h}=0.1(a=b)$. This is the reason why there are different starting locations for different $b / \theta_{h}$. 
The second visible tendency observable in Fig. 6 and Fig. 7 is that for the specified $\theta_{\sqrt{ }} / b$, the values of $D_{p}$ decrease with an increase in $b / \theta_{h}$. Moreover, for established $\theta_{v} / b$ and $b / \theta_{h}, D_{p}$ decreases with an increase in the foundation length $a\left(a / \theta_{h}\right)$. The results shown in Fig. 6 and Fig. 7 cover almost all cases that may occur in the standard sizes of shallow foundations and recently reported fluctuation scales (e.g., Pieczyńska-Kozłowska et al., 2017, Bagińska et al., 2018).

Results shown in Fig. 6 and Fig. 7 are represented as functions of $b / \theta_{h}$ and $a / \theta_{h}$. However, in the case of practical applications, the determination of the horizontal fluctuation scale is difficult. Moreover, this is still the open question: how to determine the horizontal fluctuation scale when limited number of soil soundings are accessible? For this reason, the calculations for $\theta_{h}=\infty$ were performed for different $\theta_{v} / b$ ratios. By taking an infinite value of the horizontal fluctuation scale, the horizontal axis from Fig. 6 and Fig. 7 has no sense; therefore, as a horizontal axis, a ratio of $a / \theta_{v}$ is chosen. As it can be seen in Fig. 8, the values of $D_{p}$ decrease slightly with an increase in $a / \theta_{v}$. This phenomenon is caused by changes in foundation shape. The results shown in Fig. 8 have to be treated as conservative estimations of $D_{p}$ (higher than for $\left.\theta_{h}<\infty\right)$. The similar dependence of $D_{p}$, like in Fig. 6 and Fig. 7, is observed in Fig. 8.

\subsection{Example of usage of graphs and accuracy verification}

To demonstrate the possible usage of the graphs in Fig. 6, Fig. 7 and Fig. 8, the undrained bearing capacity coefficient of variation for a set of scenarios is estimated based on these figures. To verify the outcomes, the same scenarios were analysed by the proposed algorithm individually. Finally, the obtained results are compared and juxtaposed in Table 2. The locations of the considered scenarios are shown in Fig. 9 and Fig. 10. Let's discuss the first row of Table 2. According to the initial data for the first scenario, the following dimensionless coordinates of point 1 can be found: $\theta_{v} / b=0.3, b / \theta_{h}=0.167$ and $a / \theta_{h}=0.167$. After positioning point 1 , the value $D_{p}$ of 0.685 can be read. Finally, by multiplying it by the corresponding $\mathrm{COV}_{c_{u}}$, a value of searching undrained bearing capacity coefficient of variation is obtained as 0.41 . In the last two rows, the values of $\operatorname{COV}_{c_{u}}$ and $\theta_{v}$ are taken from the study of Pieczyńska-Kozłowska et al. (2017).

According to Table 2, the obtained differences are below $10 \%$ and they are caused by the finite number of considered scenarios and interpolation errors. Note that

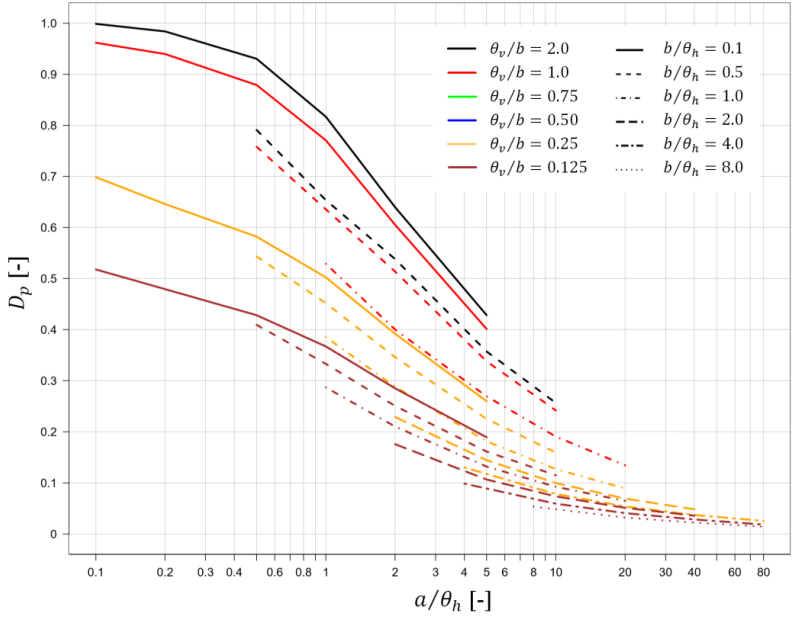

Figure 6: Graph for reading $\boldsymbol{D}_{p}$ value for ratios $\boldsymbol{\theta}_{v} / \boldsymbol{b} \in[\mathbf{2 . 0 , 1 . 0}]$ and $\boldsymbol{\theta}_{v} / \boldsymbol{b} \in[0.25,0.125]$. Note that the colour in the legend indicates $\boldsymbol{\theta}_{v} /$ $\boldsymbol{b}$ and the line type (coloured on black in the legend) indicates $\boldsymbol{\theta}_{v} / \boldsymbol{b}$.

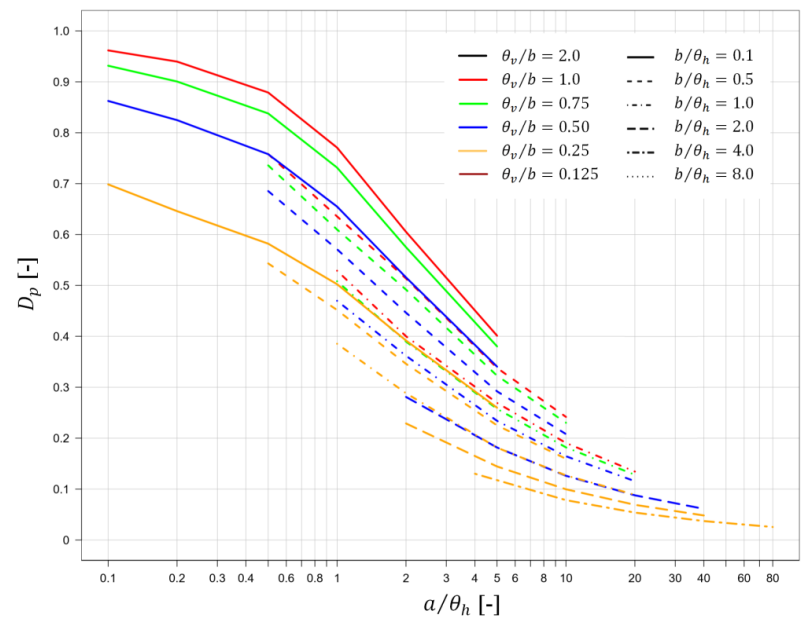

Figure 7: Graph for reading $\boldsymbol{D}_{p}$ value for ratios $\boldsymbol{\theta}_{v} / \boldsymbol{b} \in[\mathbf{1 . 0 , 0 . 2 5}$. Note that the colour in the legend indicates $\boldsymbol{\theta}_{v} / \boldsymbol{b}$ and the line type (coloured on black in the legend) indicates $\boldsymbol{\theta}_{v} / \boldsymbol{b}$.

the positioning of scenarios from Table 1 is made without the use of drafting equipment. This level of accuracy can be accepted for the approximate estimations of undrained bearing capacity coefficient of variations. Note that the errors in estimation of the fluctuation scale and $\mathrm{COV}_{c_{u}}$ may be of the same order.

\section{Summary and concluding remarks}

This paper presents a very efficient algorithm for the three-dimensional analysis of the undrained bearing capacity of shallow foundations in the case of spatially variable undrained shear strength. The algorithm uses 
Table 2: Exemplary usage of the graphs proposed in this study. Detailed descriptions are in the text.

\begin{tabular}{|c|c|c|c|c|c|c|c|c|}
\hline \multirow[t]{2}{*}{ No. } & \multicolumn{5}{|c|}{ Scenario description } & \multirow{2}{*}{$\begin{array}{l}\mathrm{COV}_{p} \text { read from } \\
\text { graphs [-] }\end{array}$} & \multirow{2}{*}{$\begin{array}{l}\operatorname{COV}_{p} \text { determined by } \\
\text { numerical analyses [-] }\end{array}$} & \multirow{2}{*}{$\begin{array}{l}\text { Difference } \\
\text { [\%] }\end{array}$} \\
\hline & $a[\mathrm{~m}]$ & $b[\mathrm{~m}]$ & $\boldsymbol{\theta}_{v}[\mathrm{~m}]$ & $\boldsymbol{\theta}_{h}[\mathrm{~m}]$ & $\operatorname{COV}_{c}[-]$ & & & \\
\hline 1 & 2.0 & 2.0 & 0.6 & 12.0 & 0.6 & 0.41 & 0.435 & $-6.1 \%$ \\
\hline 2 & 10.0 & 1.5 & 1.0 & 5.0 & 0.4 & 0.226 & 0.210 & $+7.1 \%$ \\
\hline 3 & 20.0 & 0.9 & 0.8 & 3.0 & 1.0 & 0.29 & 0.311 & $-7.2 \%$ \\
\hline 4 & 25.0 & 1.8 & 1.2 & 1.2 & 0.5 & 0.057 & 0.052 & $+8.7 \%$ \\
\hline 5 & 2.0 & 1.0 & 1.5 & 2.0 & 0.7 & 0.451 & 0.458 & $-1.5 \%$ \\
\hline 6 & 25.0 & 3.0 & 0.5 & 10.0 & 1.0 & 0.29 & 0.280 & $+3.4 \%$ \\
\hline 7 & 3.0 & 3.0 & 0.40 & $\infty$ & 0.24 & 0.13 & 0.130 & $0.0 \%$ \\
\hline 8 & 20.0 & 1.0 & 0.47 & $\infty$ & 0.51 & 0.40 & 0.403 & $-0.7 \%$ \\
\hline
\end{tabular}

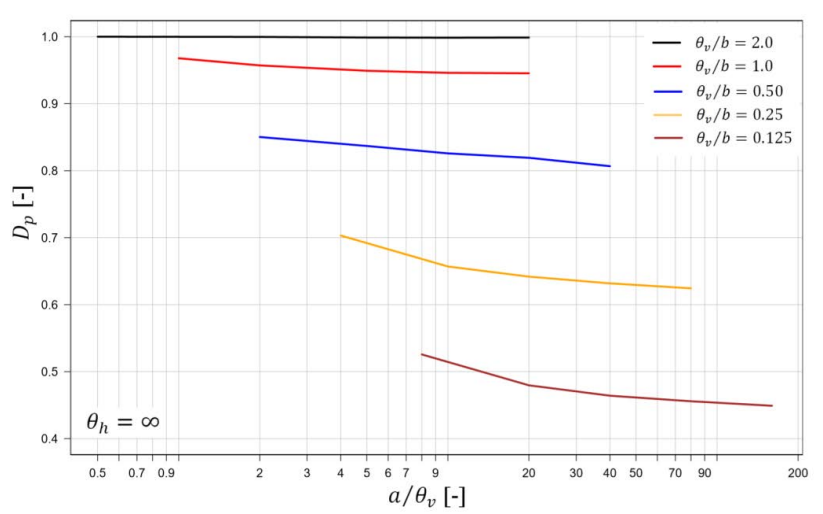

Figure 8: Graph for reading $\boldsymbol{D}_{p}$ value for an infinite horizontal fluctuation scale.

constant covariance matrix approach and is based on the Vanmarcke's spatial averaging in conjunction with upper bound approach (a kinematical failure mechanism is used). The algorithm was used in the study to analyse a series of scenarios. The objective of this extensive analysis is to create graphs that allow reading the approximate value of undrained bearing capacity coefficient of variation. The ranges of foundation geometries and fluctuation scales are assumed in a manner to cover almost all scenarios possible to exist for standard shallow foundations' geometries and for the reported values of fluctuation scales. Square and rectangular foundations were analysed. The proposed algorithm is very efficient; namely, one three-dimensional bearing capacity evaluation for spatially variable undrained shear strength takes below 1s for one core of a standard notebook. According to the presented algorithm and performed numerical analyses, the following conclusions can be drawn:

1. As indicated in Fig. 4, the resulting coefficient of variation of undrained bearing capacity obtained

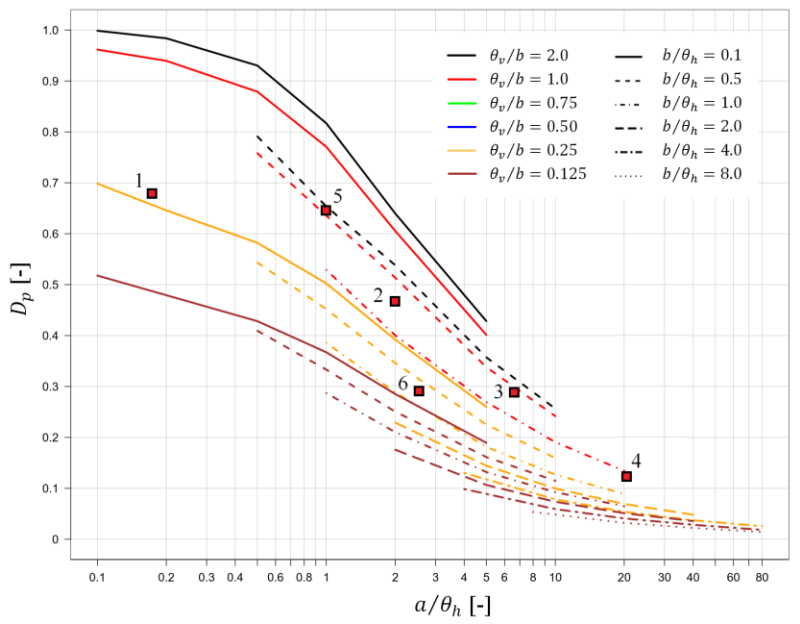

Figure 9: Illustration of example scenarios. Note that the colour in the legend indicates $\boldsymbol{\theta}_{v} / \boldsymbol{b}$ and the line type (coloured on black in the legend) indicates $\boldsymbol{\theta}_{\boldsymbol{v}} / \boldsymbol{b}$.

by using constant covariance matrix (the matrix computed for the expected value of undrained shear strength) is very close to the values obtained for individually determined covariance matrix (Chwała, 2019a). As a result of using the proposed approach, a similar accuracy can be achieved, but the numerical efficiency is improved dramatically (the computation time for one simulation is reduced about 100 times). The observed efficiency is far better than the observed for the methods based on finite element method, or finite difference method (Kawa and Puła, 2019). The problem of poor numerical efficiency is the reason why there is a lack of studies concerning threedimensional bearing capacity for spatially variable soil. The paper by Kawa and Puła is one of the first in this area. Nevertheless, the efficiency of the method 


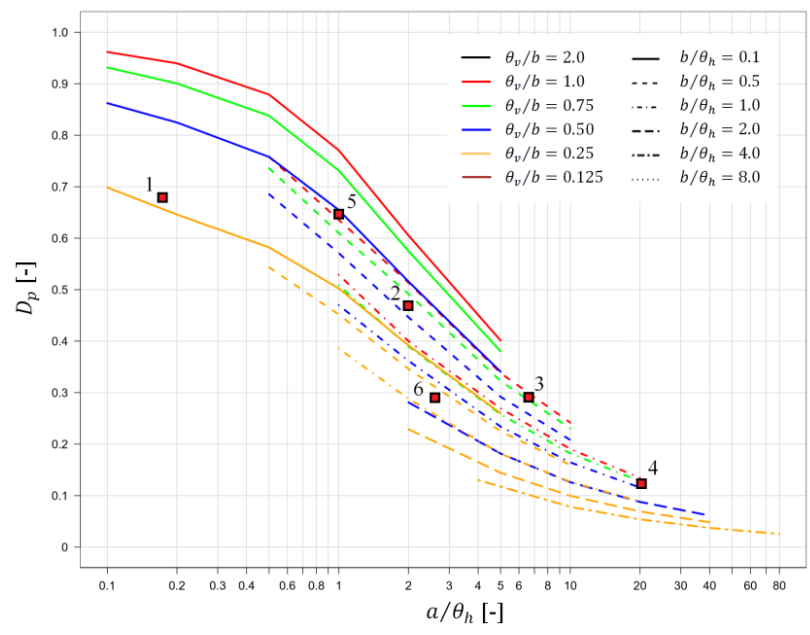

Figure 10: Illustration of example scenarios. Note that the colour in the legend indicates $\boldsymbol{\theta}_{v} / \boldsymbol{b}$ and the line type (coloured on black in the legend) indicates $\boldsymbol{\theta}_{v} / \boldsymbol{b}$.

proposed in this study allows for its usage for the applications that required many simulations to be analysed, like the analyses performed in this study or the recently proposed approach for searching optimal borehole locations (Chwała, 2019b).

2. The proposed algorithm was used to create graphs that allow fast reading of approximate coefficient of variation of undrained bearing capacity. The usage of the graphs is demonstrated in the study, the obtained results indicate that the final accuracy can be accepted for this purpose. The graphs can be used if vertical fluctuation scale and coefficient of variation of undrained shear strength are known. However, those values can be relatively easily determined from CPT sounding. The graphs can be used to make a comparison with the other three-dimensional methods for random bearing capacity estimation. As it can be observed in Fig. 6, Fig. 7 and Fig. 8, there are no worst-case scenario (e.g., Fenton and Griffiths, 2008; Ching et. al., 2017) in the coefficients of variation of bearing capacity exists. This is in agreement with earlier experiences where the worst-case is observed mostly in mean values (e.g., Puła et al., 2017).

3. The study indicates the importance of performing three-dimensional analyses if the soil spatial variability is taken into account. A three-dimensional algorithm is necessary to consider realistically the soil spatial variability that has a three-dimensional nature. This phenomenon is visible in Fig. 6 and Fig. 7, where a significant decreasing tendency in variation coefficient of undrained bearing capacity is observed with an increase of foundation length $a$. Results indicate that if two-dimensional analysis is assumed, the resulting estimation of COVp will be a conservative one.

Acknowledgements: The author is grateful for the support by National Science Centre (grant: Miniatura 2; Project No. 2018/02/X/ST8/02939)

\section{References}

[1] Ali A, Lyamin AV, Huang J, Sloan SW, Cassidy MJ. Effect of Spatial Correlation Length on the Bearing Capacity of an Eccentrically Loaded Strip Footing. $6^{\text {th }}$ APSSRA 2016, 311-316.

[2] Bagińska I, Kawa M, Janecki W. Estimation of spatial variability properties of mine waste dump using CPTu results - case study. Proceedings of the 4th International Symposium on Cone Penetration Testing (CPT'18), Delft, The Netherlands, 21-22 June 2018; eds. Michael A. Hicks, Federico Pisanò, Joek Peuchen. Leiden : CRC Press/Balkema, cop. 2018. s. 109-115.

[3] Chen F, Wang L, Zhang W. Reliability assessment on stability of tunnelling perpendicularly beneath an existing tunnel considering spatial variabilities of rock mass properties. Tunnelling and Underground Space Technology, 88 (2019), 276289. https://doi.org/10.1016/j.tust.2019.03.013

[4] Ching J, Wu TJ, Stuedlein AW, Bong T. Estimating horizontal scale of fluctuation with limited CPT soundings. Geoscience Frontiers, 2018; Volume 9, Issue 6, 1597-1608. https://doi. org/10.1016/j.gsf.2017.11.008

[5] Ching J, Phoon KK, Sung SP. Worst case scale of fluctuation in basal heave analysis involving spatial variable clays, Structural Safety, 68 (2017), pp. 28-42

[6] Ching J, Phoon KK, Sung SP. Worst case scale of fluctuation in basal heave analysis involving spatially variable clays. Structural Safety, Vol. 68, 28-42. https://doi.org/10.1016/j. strusafe.2017.05.008

[7] Chwała M. (2019a). Undrained bearing capacity of spatially random soil for rectangular footings. Soils and Foundations 59 (2019) 1508-1521. https://doi.org/10.1016/j.sandf.2019.07.005

[8] Chwała M. (2019b). Bearing Capacity for Spatially Random Soil Considering Cone Penetration Test Locations. In: Ching, J., Li, D. Q. \& Zhang, J., (Eds), Proceedings, 7th International Symposium on Geotechnical Safety and Risk, Research Publishing, 2019, 11-13 December, 2019, Taipei, Taiwan, 899pp. ISBN : 978-98111-2725-0, DOI : 10.3850/9789811127250-toc.

[9] Fenton GA, Griffiths DV. Risk assessment in geotechnical engineering. Wiley; 2008.

[10] Goh Anthony TC , Zhang WG, Wong KS. Deterministic and reliability analysis of basal heave stability for excavation in spatial variable soils. Computers and Geotechnics, 108 (2019), 152-160. https://doi.org/10.1016/j.compgeo.2018.12.015

[11] Gourvenec S, Randolph M, Kingsnorth O. Undrained Bearing Capacity of Square and Rectangular Footings. International Journal of Geomechanics, 2006; Vol. 6, 3, 147-157.

[12] Horn RA, Johnson CR. Matrix Analysis. Cambridge University Press, 1985. 
[13] Huang J, Lyamin AV, Griffiths DV, Sloan SW, Krabbenhoft K, Fenton GA. Undrained bearing capacity of spatially random clays by finite elements and limit analysis. Proceedings of the 18th ICSMGE, Paris (2013), pp. 731-734

[14] Johari A, Hosseini SM, Keshavarz. Reliability analysis of seismic bearing capacity of strip footing by stochastic slip lines method. Computers and Geotechnics, 91 (2017), 203-217. https://doi.org/10.1016/j.compgeo.2017.07.019

[15] Kawa M, Puta W. 3D bearing capacity probabilistic analyses of footings on spatially variable $c-\varphi$ soil. Acta Geotechnica, June 2019. DOI: $10.1007 / \mathrm{s} 11440-019-00853-3$

[16] Kirkpatrick S, Gelatt CD, Vecchi MP. Optimization by Simulated Annealing. Science, 1983; 220, 671-680.

[17] Kirkpatrick S. Optimization by Simulated Annealing: Quantitative Studies. Journal of Statistical Physics, 1984; Vol. 34, Nos. 5/6.

[18] Lu Q, Xiao Z, Zheng J, Shang Y.Probabilistic assessment of tunnel convergence considering spatial variability in rock mass properties using interpolated autocorrelation and response surface method. Geoscience Frontiers, 9(6), 2018, 1619-1629. https://doi.org/10.1016/j.gsf.2017.08.007

[19] Nuttall JD. Estimation of Horizontal and Vertical Scales of Fluctuation from CPT Data Using Neural Networks and Random Fields. In: Ching, J., Li, D. Q. \& Zhang, J., (Eds), Proceedings, 7th International Symposium on Geotechnical Safety and Risk, Research Publishing, 2019, 11-13 December 2019, Taipei, Taiwan, 899pp. ISBN : 978-981-11-2725-0, DOI : 10.3850/9789811127250-toc.

[20] Pan Q, Dias D.Probabilistic Analysis of a Rock Tunnel Face Using Polynomial Chaos Expansion Method[J]. International Journal of Geomechanics, 2018, 18(4): 04018013. https://doi. org/10.1061/(ASCE)GM.1943-5622.0001116.

[21] Pieczyńska-Kozłowska JM. Comparison between two methods for estimating the vertical scale of fluctuation for modelling random geotechnical problems. Studia Geotechnica et Mechanica 37(2015), 4:95-103.

[22] Pieczyńska-Kozłowska JM, Puła W, Griffiths DV, Fenton GA. Influence of embedment, self-weight and anisotropy on bearing capacity reliability using the random finite element method. Computers and Geotechnics, 2015; 67, 229-238.

[23] Pieczyńska-Kozłowska JM, Puła W, Vessia G. A collection of fluctuation scale values and autocorrelation functions of fine deposits in Emilia Romagna Palin, Italy; Geo-Risk 2017; Reston: American Society of Civil Engineers, 290-299.

[24] Puta W. On some aspects of reliability computations in bearing capacity of shallow foundations. In: Griffiths DV, Fenton Gordon A, editors. Puta in: probabilistic methods in geotechnical engineering. CISM courses and lectures, Wien, New York: Springer, 2007; No. 491, 127-45.

[25] Puła W, Chwata M. On spatial averaging along random slip lines in the reliability computations of shallow strip foundations. Computers and Geotechnics, 2015; 68, 128-136. https://doi. org/10.1016/j.compgeo.2015.04.001

[26] Puła W, Pieczyńska-Kozłowska JM, Chwała M. Search for the Worst-Case Correlation Length in the Bearing Capacity Probability of Failure Analyses; Geo-Risk 2017; Reston: American Society of Civil Engineers, 534-544.
[27] Puta W, Chwała M. Random bearing capacity evaluation of shallow foundations for asymmetrical failure mechanisms with spatial averaging and inclusion of soil self-weight. Computers and Geotechnics, 2018; 101, 176-195. https://doi. org/10.1016/j.compgeo.2018.05.002

[28] Simoes JT, Neves LC, Antao AN, Guerra NMC. Probabilistic analysis of bearing capacity of shallow foundations using three-dimensional limit analyses. Int. J. Comput. Methods, 11 (02) (2014) pp. 1342008-1-20

[29] Vanmarcke EH. Random fields - analysis and synthesis. Cambridge: MIT Press, 1983. 


\section{Appendix A}

The bearing capacity formula for rough foundation base according to the study by Chwała (2019a) can be expressed by Eq. (A.1):

$p=p_{1}+p_{2}+p_{3}+p_{4}$

where, $p_{1}, p_{2}, p_{3}$ and $p_{4}$ are contributions to the bearing capacity for the bottom side, the top side, the left side and the right side of the failure mechanism, respectively (see Fig. 1):

$$
\begin{aligned}
& p_{1}=b_{2}\left(a-\left(d_{1}+d_{2}\right)\right) m_{1}+0.5 b_{2} d_{1} n_{1} m_{2}+0.5 b_{2} d_{2} n_{2} m_{3} \\
& p_{2}=b_{1}\left(a-\left(d_{1}+d_{2}\right)\right) m_{4}+0.5 b_{1} d_{1} n_{3} m_{5}+0.5 b_{1} d_{2} n_{4} m_{6} \\
& p_{3}=0.5 b_{1} d_{1} n_{5} m_{7}+0.5 b_{2} d_{1} n_{6} m_{8} \\
& p_{4}=0.5 b_{1} d_{2} n_{7} m_{9}+0.5 b_{2} d_{2} n_{8} m_{10}
\end{aligned}
$$

Coefficients $m_{i}$ and $n_{i}$ are juxtaposed in Table A.1.
Table A.1: Coefficients from Eq. (A.2)-Eq. (A.5) for rough and smooth foundation base. Note that the undrained shear strengths $c_{i}$ are defined individually for each dissipation region (for more details see Chwała, 2019a).

\section{Coeff. Expression}

$$
\begin{aligned}
& m_{1} \quad c_{1} \cot \beta_{2}+2 c_{21}\left(\alpha_{2}+\beta_{2}\right)+c_{2} \cot \alpha_{2} \\
& m_{2} \quad c_{6} \cot \alpha_{2}+2 c_{24}\left(\alpha_{2}+\beta_{2}\right)+c_{5} \cot \beta_{2} \\
& m_{3} \quad c_{8} \cot \alpha_{2}+2 c_{23}\left(\alpha_{2}+\beta_{2}\right)+c_{7} \cot \beta_{2} \\
& m_{4} \quad c_{3} \cot \beta_{3}+2 c_{22}\left(\alpha_{3}+\beta_{3}\right)+c_{4} \cot \alpha_{3} \\
& m_{5} \quad c_{10} \cot \alpha_{3}+2 c_{26}\left(\alpha_{3}+\beta_{3}\right)+c_{9} \cot \beta_{3} \\
& m_{6} \quad c_{12} \cot \alpha_{3}+2 c_{25}\left(\alpha_{3}+\beta_{3}\right)+c_{11} \cot \beta_{3} \\
& m_{7} \\
& c_{16} \cot \alpha_{1}+2 c_{28}\left(\alpha_{1}+\beta_{1}\right)+c_{14} \cot \beta_{1} \\
& m_{8} \\
& c_{15} \cot \alpha_{1}+2 c_{27}\left(\alpha_{1}+\beta_{1}\right)+c_{13} \cot \beta_{1} \\
& m_{9} \\
& c_{20} \cot \alpha_{4}+2 c_{30}\left(\alpha_{4}+\beta_{4}\right)+c_{19} \cot \beta_{4} \\
& m_{10} \\
& c_{18} \cot \alpha_{4}+2 c_{29}\left(\alpha_{4}+\beta_{4}\right)+c_{17} \cot \beta_{4} \\
& n_{1} \\
& n_{2} \\
& n_{3} \\
& n_{4} \\
& n_{5} \\
& n_{6} \\
& n_{7} \\
& n_{8} \\
& \sqrt{1+\frac{b_{2}^{2}}{d_{1}^{2}\left(\sin \beta_{2}\right)^{2}}} \\
& \sqrt{1+\frac{b_{2}^{2}}{d_{2}^{2}\left(\sin \beta_{2}\right)^{2}}} \\
& \sqrt{1+\frac{b_{1}^{2}}{d_{1}^{2}\left(\sin \beta_{3}\right)^{2}}} \\
& \sqrt{1+\frac{b_{1}^{2}}{d_{2}^{2}\left(\sin \beta_{3}\right)^{2}}} \\
& \sqrt{1+\frac{d_{1}^{2}}{b_{1}^{2}\left(\sin \beta_{1}\right)^{2}}} \\
& \sqrt{1+\frac{d_{1}^{2}}{b_{2}^{2}\left(\sin \beta_{1}\right)^{2}}} \\
& \sqrt{1+\frac{d_{2}^{2}}{b_{1}^{2}\left(\sin \beta_{4}\right)^{2}}} \\
& \sqrt{1+\frac{d_{2}^{2}}{b_{2}^{2}\left(\sin \beta_{4}\right)^{2}}}
\end{aligned}
$$

